\title{
Semiclassical effects in black hole interiors
}

\author{
William A. Hiscock* and Shane L. Larson ${ }^{\dagger}$ \\ Department of Physics, Montana State University, Bozeman, Montana 59717 \\ Paul R. Anderson \\ Department of Physics, Wake Forest University, Winston-Salem, North Carolina 27109
}

(Received 2 January 1997; revised manuscript received 23 May 1997)

\begin{abstract}
First-order semiclassical perturbations to the Schwarzschild black hole geometry are studied within the black hole interior. The source of the perturbations is taken to be the vacuum stress-energy of quantized scalar, spinor, and vector fields, evaluated using analytic approximations developed by Page and others (for massless fields) and the DeWitt-Schwinger approximation (for massive fields). Viewing the interior as an anisotropic collapsing cosmology, we find that minimally or conformally coupled scalar fields, and spinor fields, decrease the anisotropy as the singularity is approached, while vector fields increase the anisotropy. In addition, we find that for massless fields of all spins, the massive conformally coupled scalar field, and massive vector fields, the metric perturbations initially strengthen the singularity, while for minimally coupled massive scalar and spinor fields the metric perturbations tend to initially slow the growth of curvature. [S0556-2821(97)06118-3]
\end{abstract}

PACS number(s): 04.70.Dy, 04.60.Gw, 04.62.+v

\section{INTRODUCTION}

The application of quantum field theory to curved space has resulted in a large array of interesting and important results. These include black hole evaporation [1] and its implications for black hole thermodynamics [2], the dissipation of anisotropy by particle production in cosmological spacetimes [3-11], and the removal of cosmological singularities by vacuum polarization effects [12-16]. One of the places for which quantum effects have been studied the least is the interior of a black hole. One might think that such studies are not interesting because no observer from the exterior region can probe the interior region unless they choose to fall into the hole. However the existence of black hole evaporation makes it quite possible to eventually learn about quantum effects in the interior of a black hole. ${ }^{1}$ This is because as a black hole evaporates more and more of its interior is exposed. Thus not only can quantum effects in the interior of a black hole eventually be detected, they may have a significant influence on the evaporation process.

Quantum effects in the interior may in fact have a direct bearing on two of the most fundamental outstanding issues relating to the quantum mechanics of black holes. One of these is the question of what happens during the late stages of black hole evaporation, that is, what is the end point of the evaporation process? The other is the question of what happens to the information about how the black hole formed. There are at least two ways in which quantum effects in the interior could affect the answers to these questions. One is that if quantum effects remove the singularity predicted by general relativity then it is very likely that the evolution will be unitary and information will not be destroyed. A second

\footnotetext{
*Electronic address: billh@orion.physics.montana.edu.

${ }^{\dagger}$ Electronic address: shane@physics.montana.edu.

‡Electronic address: paul@planck.wfu.edu.

${ }^{1}$ By interior we mean here the region inside the apparent horizon.
}

possibility is that quantum effects could cause the evaporation process to cease, leaving a zero temperature black hole remnant. If the remnant has an event horizon the information would very likely be trapped inside the black hole. Since the temperature of a black hole is determined by the surface gravity at its horizon and since the evaporation process causes the horizon to be at points which were previously in the (apparent) interior, it is clear that the geometry of the interior is likely to influence the evaporation process as it progresses.

One interesting quantum effect that seems likely to occur inside the horizon of a black hole is the dissipation of anisotropy and possibly inhomogeneity due to particle production. This is because the interior of such a black hole can be thought of as an anisotropic and possibly inhomogeneous cosmology. For example the interior of a Schwarzschild black hole can be thought of as a homogeneous, anisotropic cosmology of the Kantowski-Sachs family [17]. It has been well established that particle production dissipates anisotropy in Bianchi type I spacetimes [3-11]. If the process of anisotropy dissipation occurs it will certainly alter the geometry in the interior of a black hole.

For these reasons it is interesting to examine quantum effects in the interior of a black hole. To do so for either the interior or exterior of an evaporating black hole would be an enormously difficult task at present due to problems that one would encounter in computing the stress-energy tensors for quantized fields in the relevant spacetime. However, computing the stress-energy tensors for these fields in the case of a spherically symmetric black hole in thermal equilibrium with radiation in a cavity, i.e., with the fields in the HartleHawking state, is a much more tractable problem. The reason is that there are then three Killing vector fields in the spacetime, which makes the mode equations separable.

For a black hole in equilibrium with fields in the HartleHawking state, analytical approximations for the stressenergy tensors of various types of quantized fields have been obtained. The derivations of most of these approximations 
have been for the exterior region, but, as is discussed later, they all can easily be extended to the interior region. These approximations include those of Page, Brown, and Ottewill [18-20] for conformally invariant fields in Schwarzschild spacetime, that of Frolov and Zel'nikov [21] for conformally invariant fields in a general static spacetime, that of Anderson, Hiscock, and Samuel [22] for massless arbitrarily coupled scalar fields in a general static spherically symmetric spacetime, and the DeWitt-Schwinger approximation for massive fields which was derived by Frolov and Zel'nikov $[23,24]$ for Kerr spacetime, by Anderson, Hiscock, and Samuel [22] for a scalar field in a general static spherically symmetric spacetime and most recently by Herman and Hiscock [25] for an arbitrary spacetime.

In this paper the various approximations mentioned above are used to investigate quantum effects in the interior of a Schwarzschild black hole when the fields are in the HartleHawking state. The resulting semiclassical backreaction equations are linearized about the classical geometry and their solutions are found. The questions of whether backreaction effects tend to isotropize the spacetime and whether they tend to "soften" the geometry as the singularity is approached are addressed. Although the questions of whether the anisotropy is completely dissipated or whether the singularity is removed cannot be answered by examining linear perturbations, the results do provide insight into these issues.

In Sec. II the interior geometry of a Schwarzschild black hole is reviewed and in Sec. III the various analytical approximations are reviewed and discussed. Solutions to the linearized backreaction equations which are derived using these approximations are displayed in Sec. IV. In Sec. V the dissipation of anisotropy is computed and in Sec. VI the change in the curvature is computed. The results are summarized and discussed in Sec. VII.

\section{SCHWARZSCHILD BLACK HOLE INTERIOR}

The Schwarzschild black hole is described by the metric

$$
d s^{2}=-\left(1-\frac{2 M}{r}\right) d t^{2}+\left(1-\frac{2 M}{r}\right)^{-1} d r^{2}+r^{2} d \Omega^{2},
$$

where $d \Omega^{2}$ is the metric of the two-sphere. The coordinate $r$ runs from 0 to $\infty$, and $t$ from $-\infty$ to $+\infty$. We are thus considering the complete Schwarzschild manifold, as is appropriate with the Hartle-Hawking vacuum state. The black hole interior is the region in which $0 \leqslant r \leqslant 2 M$. In the interior, the vector field $\partial / \partial r$ is timelike and the vector field $\partial / \partial t$ is spacelike; hence, the coordinate $t$ is a spatial coordinate, while $r$ is a time coordinate.

The nature of the interior is more easily visualized if new coordinate names are adopted to reflect the physical nature of the coordinates in the region of interest. Defining new coordinates by setting

$$
T \equiv r, \quad x \equiv t,
$$

the metric takes the form

$$
d s^{2}=-\left(\frac{2 M}{T}-1\right)^{-1} d T^{2}+\left(\frac{2 M}{T}-1\right) d x^{2}+T^{2} d \Omega^{2} .
$$

The metric given by Eq. (3) is clearly an anisotropic homogeneous cosmology. The vector field $\partial / \partial t$ is, in the interior, one of the spacelike Killing vector fields (along with those on the two-sphere) which guarantee spatial homogeneity. The spatial coordinate $x$ here runs from $-\infty$ to $+\infty$, while $T$ runs from $2 M$ down to zero at the curvature singularity in the black hole interior.

The Schwarzschild manifold contains both an anisotropic expanding universe, the "white hole" portion of the extended geometry, and an anisotropic collapsing universe, the black hole interior. In this paper we shall base our discussion on the black hole interior portion of the geometry, but all conclusions may be restated in terms of the expanding white hole geometry due to the time reversal symmetry of both the Schwarzschild geometry and the Hartle-Hawking state we shall use to perturb it. However, the boundary conditions for the fields in the two cases are very different. In the black hole case they are "initial" conditions, while in the white hole case they are "final" conditions for the interior region.

While it is conventional to write homogeneous cosmological metrics in terms of a proper time coordinate, i.e.,

$$
\tau=\int \frac{d T}{\left(\frac{2 M}{T}-1\right)^{1 / 2}},
$$

in the present case the spatial metric components cannot be expressed in closed algebraic form in terms of such a coordinate. Upon carrying out the integral in Eq. (4), one finds that the range of the coordinate $T$ from $2 M$ down to 0 corresponds to an interval of proper time equal to $\pi M$.

The spacetime described by the metric of Eq. (3), viewed as a cosmological model, is an anisotropic but homogeneous spacetime in which (as $T$ proceeds from $2 M$ down to zero) two spatial dimensions are collapsing while one is expanding. The interior Schwarzschild cosmology is a special case of a type I Kantowski-Sachs model [17].

Since the Schwarzschild metric is a vacuum solution, there is no naturally defined four-velocity of cosmological "'matter;"' however, to explore the properties of the solution as an anisotropic cosmology, it is helpful to define a set of fiducial geodesic observers with four-velocities given by

$$
u^{\alpha}=\left(\left(\frac{2 M}{T}-1\right)^{1 / 2}, 0,0,0\right) \text {. }
$$

These observers travel along world lines with $x, \theta$, and $\phi$ constant. In terms of the conserved quantities normally used to describe geodesics in the exterior Schwarzschild metric, these observers have zero angular momentum and zero energy at infinity.

The proper volume of a cube defined by a set of fiducial observers at the corners, separated by coordinate distances $\Delta x, \Delta \theta$, and $\Delta \phi$ is given by

$$
V(T)=\left(\frac{2 M}{T}-1\right)^{1 / 2} T^{2} \Delta x \Delta \theta \Delta \phi .
$$

Since the fiducial observers have four-velocities given by Eq. (5), the quantities $\Delta x, \Delta \theta$, and $\Delta \phi$ are constant. The volume goes to zero at both $T=0$ and $T=2 M$. 
Near the singularity at $T=0$, the Schwarzschild metric of Eq. (3) may be put into a form which is locally asymptotic to a Kasner universe. Let coordinates $y$ and $z$ be defined as functions of and locally in the neighborhood of a point $\left(\theta_{0}, \phi_{0}\right)$ by

$$
y=2 M\left(\theta-\theta_{0}\right), \quad z=2 M \sin \left(\theta_{0}\right)\left(\phi-\phi_{0}\right) .
$$

While these coordinates cannot be extended to cover the two-sphere they are perfectly adequate to describe the expansion and contraction of the cosmology in a local neighborhood. Near the singularity, the Schwarzschild metric then takes the form of a Kasner universe with exponents $p_{1}=-1 / 3, p_{2}=p_{3}=2 / 3$ :

$$
d s^{2}=-d \tau^{2}+\left(\frac{\tau}{\tau_{0}}\right)^{-2 / 3} d x^{2}+\left(\frac{\tau}{\tau_{0}}\right)^{4 / 3}\left(d y^{2}+d z^{2}\right)
$$

where $\tau_{0}=4 M / 3$ and $\tau=\left(2 T^{3} / M\right)^{1 / 2} / 3$. In a similar fashion, the metric may be approximated by a flat Kasner $\left(p_{1}=1, p_{2}=p_{3}=0\right)$ solution near $T=2 M$. There the cosmological proper time has the asymptotic form $\tau=4 M(1-T / 2 M)^{1 / 2}$, and the asymptotic form of the metric is

$$
d s^{2}=-d \tau^{2}+\frac{\tau^{2}}{16 M^{2}} d X^{2}+\left(d y^{2}+d z^{2}\right)
$$

as $\tau \rightarrow 0$. The singular behavior of Eq. (9) is of course only apparent; the surface $\tau=0$ is actually the black hole event horizon.

\section{APPROXIMATE STRESS-ENERGY TENSORS}

\section{A. Massless fields}

To calculate the linearized metric perturbations to the Schwarzschild geometry resulting from the presence of quantized fields, it is necessary to know the values of the stress-energy tensors of those fields. Calculating the stressenergy tensor for a quantized field on a black hole background spacetime is an arduous task, which has been carried to completion only for a few cases. Howard and Candelas have computed the stress-energy of a conformally invariant scalar field in the Schwarzschild geometry [26,27]. Jensen and Ottewill have computed the vacuum stress-energy of a massless vector field in the Schwarzschild geometry [28]. More recently Anderson, Hiscock, and Samuel have developed a method for computing the vacuum stress-energy for a general (arbitrary curvature coupling and mass) scalar field in an arbitrary static spherical spacetime and have applied their method to the Reissner-Nordström geometry [22,29].

In each of these studies, an analytic expression for $\left\langle T_{\mu \nu}\right\rangle$ has been developed as a consequence of the procedure used to compute the exact values for $\left\langle T_{\mu \nu}\right\rangle$. These approximate expressions are generated by using a fourth order WKB expansion for the field modes in the unrenormalized expression for $\left\langle T_{\mu \nu}\right\rangle$ and then subtracting off the DeWitt-Schwinger counterterms [30] to renormalize the stress-tensor. The resulting analytic expressions are closely related to approximate expressions for the vacuum stress-energy derived by Page, Brown, and Ottewill (PBO) [18-20] and Frolov and Zel'nikov (FZ) [21]. The analytic approximation found by
Howard and Candelas is identical to the PBO approximation for the conformal scalar field's stress-energy in Schwarzschild spacetime; further, their numerical results show that the approximation is quite accurate for all values of $r$ down to the horizon. In the case of the vector field, the analytic expression derived by Jensen and Ottewill is equal to the PBO approximation for a conformal vector field plus a traceless term proportional to $r^{-4}$; the resulting expression yields a good match to the numerical results for the vector field [28]. The analytic approximation developed by Anderson, Hiscock, and Samuel reduces to the FZ approximation when restricted to conformal coupling; it has generally been shown to be valid for arbitrary curvature coupling when compared to numerical results in the Reissner-Nordström geometry (which, of course, includes Schwarzschild geometry as a special case).

Each of these expressions has been derived in the exterior region of the black hole. There is good reason to believe they are valid in the interior also. The components of the curvature tensors in an orthonormal frame are analytic functions of $r$ near the event horizon. Each of the approximations is also an analytic function of the radial coordinate $r$ near the event horizon. Thus the analytic extension of these approximations into the interior region is trivial to obtain. Further Candelas and Jensen [31] have numerically computed $\left\langle\phi^{2}\right\rangle$ in the interior of a Schwarzschild black hole when the field is in the Hartle-Hawking state. They find that Page's approximation [18] for $\left\langle\phi^{2}\right\rangle$ arises in a natural way from the calculation of the renormalized Feynman Green function in the interior region and that it is a good approximation in much of the interior region.

In this paper the Anderson, Hiscock, Samuel approximate analytic stress-energy tensor will be used to describe the effects of quantized massless scalar fields with arbitrary curvature coupling in the Schwarzschild interior. The JensenOttewill analytic approximation will be used for the stressenergy tensor of massless vector fields. Massless spinor fields will be treated using the PBO approximation. It should be kept in mind, however, that the spinor field expression has not yet been tested against an accurate numerical computation to establish its validity.

The components of the stress-energy tensor in Schwarzschild coordinates may then be expressed as follows:

$$
\left\langle T_{\mu \nu}\right\rangle=C_{\mu \nu}+(\xi-1 / 6) D_{\mu \nu},
$$

where $C_{\mu \nu}$ represents the conformally invariant contribution to the vacuum stress-energy from all the fields, and $D_{\mu \nu}$ represents the nonconformal contribution due to the scalar fields, which we allow to have arbitrary curvature coupling. Applying the approximations discussed above,

$$
\begin{aligned}
C_{T}^{T}= & \frac{\epsilon}{\lambda M^{2}}\left\{a\left(1+2\left(\frac{2 M}{T}\right)+3\left(\frac{2 M}{T}\right)^{2}\right]+a_{3}\left(\frac{2 M}{T}\right)^{3}\right. \\
& \left.+a_{4}\left(\frac{2 M}{T}\right)^{4}+a_{5}\left(\frac{2 M}{T}\right)^{5}+a_{6}\left(\frac{2 M}{T}\right)^{6}\right\}
\end{aligned}
$$

where

$$
a=h(0)+\frac{7}{8} h(1 / 2)+h(1)
$$




$$
\begin{gathered}
a_{3}=4 h(0)-\frac{13}{2} h(1 / 2)-76 h(1), \\
a_{4}=5 h(0)-\frac{35}{8} h(1 / 2)+295 h(1), \\
a_{5}=6 h(0)-\frac{9}{4} h(1 / 2)-54 h(1), \\
C_{x}^{x}=\frac{3 \epsilon}{\lambda M^{2}}\left\{-a\left[1+2\left(\frac{2 M}{T}\right)^{+3}\left(\frac{2 M}{T}\right)^{2}+4\left(\frac{2 M}{T}\right)^{3}\right]\right. \\
\left.+b_{4}\left(\frac{2 M}{T}\right)^{4}+b_{5}\left(\frac{2 M}{T}\right)^{5}+b_{6}\left(\frac{2 M}{T}\right)^{6}\right\}
\end{gathered}
$$

where

$$
\begin{aligned}
& b_{4}=-5 h(0)-\frac{45}{8} h(1 / 2)+105 h(1), \\
& b_{5}=-6 h(0)-\frac{31}{4} h(1 / 2)-26 h(1), \\
& b_{6}=33 h(0)+\frac{161}{8} h(1 / 2)+83 h(1),
\end{aligned}
$$

and

$$
\begin{gathered}
C_{\theta}^{\theta}=C_{\phi}^{\phi}=\frac{\epsilon}{\lambda M^{2}}\left\{a\left[1+2\left(\frac{2 M}{T}\right)+3\left(\frac{2 M}{T}\right)^{2}\right]+c_{3}\left(\frac{2 M}{T}\right)^{3}\right. \\
\left.+c_{4}\left(\frac{2 M}{T}\right)^{4}+c_{5}\left(\frac{2 M}{T}\right)^{5}+c_{6}\left(\frac{2 M}{T}\right)^{6}\right\} \\
c_{3}=4 h(0)+\frac{17}{2} h(1 / 2)+44 h(1) \\
c_{4}=5 h(0)+\frac{85}{8} h(1 / 2)-305 h(1) \\
c_{5}=6 h(0)+\frac{51}{4} h(1 / 2)+66 h(1) \\
c_{6}=-9 h(0)+\frac{87}{8} h(1 / 2)-579 h(1)
\end{gathered}
$$

The constants $\epsilon$ and $\lambda$ are defined by $\epsilon=\hbar / M^{2}$, $\lambda=\left(45 \times 2^{13}\right) \pi^{2}$, and $h(s)$ is the number of helicity states in, respectively, the scalar, spinor, and vector fields present. Explicitly, $h(0)$ simply counts the number of scalar fields present, $h(1 / 2)$ is equal to 2 (or 4 ) for each two- (or four-) component spinor field present; $h(1)$ is equal to 2 times the number of vector fields present. The nonconformal contribution to the scalar field stress-energy is given by

$$
\begin{aligned}
D_{T}^{T}= & -60 h(0) \frac{\epsilon}{\lambda M^{2}}\left(\frac{2 M}{T}\right)^{3}\left[4-3\left(\frac{2 M}{T}\right)\right]\left[1+2\left(\frac{2 M}{T}\right)\right. \\
& \left.+3\left(\frac{2 M}{T}\right)^{2}\right], \\
D_{x}^{x}= & 180 h(0) \frac{\epsilon}{\lambda M^{2}}\left(\frac{2 M}{T}\right)^{4}\left[1+2\left(\frac{2 M}{T}\right)-5\left(\frac{2 M}{T}\right)^{2}\right] \\
D_{\theta}^{\theta}= & 120 h(0) \frac{\epsilon}{\lambda M^{2}}\left(\frac{2 M}{T}\right)^{3}\left[1+2\left(\frac{2 M}{T}\right)+3\left(\frac{2 M}{T}\right)^{2}\right. \\
& \left.-12\left(\frac{2 M}{T}\right)^{3}\right]
\end{aligned}
$$

These expressions exhibit a variety of interesting behavior in the black hole interior. The energy density, $\rho=-\left\langle T_{T}^{T}\right\rangle$, is negative at the horizon for the conformally coupled scalar field and the vector field; it is positive there, however, for the spinor field and for any scalar field with $\xi>1 / 4$. The energy density diverges negatively as the singularity is approached for all conformal fields; however, the density diverges positively for scalar fields with $\xi<5 / 36$, which includes the minimally coupled scalar field. There is a particular surface, $T=3 M / 2$, on which the energy density of the scalar field is independent of the curvature coupling.

The spatial stress in the $x$ direction, $\left\langle T_{x}^{x}\right\rangle$, is positive at the horizon for all scalar fields with $\xi<4 / 15$, which includes both the minimally coupled and conformally coupled cases, and for the conformal vector field. The stress is negative at the horizon for the spinor field. This stress diverges in a positive fashion as the singularity is approached for all conformal fields and also for the minimally coupled scalar field.

The tangential stress, $\left\langle T_{\theta}^{\theta}\right\rangle$, is everywhere positive in the domain of interest for the minimally coupled scalar field and the spinor field; it is also everywhere negative for the vector field. The conformal scalar field has $\left\langle T_{\theta}^{\theta}\right\rangle$ positive at the horizon, but diverging negatively as the singularity is approached.

\section{B. Massive fields}

The technique of choice for computing an approximate renormalized stress-energy tensor in the massive case is the DeWitt-Schwinger approximation for $\left\langle T_{\mu}^{\nu}\right\rangle$. This is obtained by performing the DeWitt-Schwinger expansion of the stress-energy tensor, in inverse square powers of the field mass, $m$, and then subtracting off the first, divergent terms of the expansion [32]. The remaining terms of the asymptotic series may be used as an analytic approximation to $\left\langle T_{\mu}^{\nu}\right\rangle$. In this paper, approximations for the stress-energy tensor of massive quantized fields have been derived from the previous work of Frolov and Zel'nikov [24], who used the DeWitt-Schwinger approximation to find the renormalized stress-energy for massive fields in the Kerr spacetime. For the massive scalar field in the Schwarzschild limit, Frolov and Zel'nikov's Kerr results have been found to reduce to the stress-energy obtained by other renormalization methods [23]. 
By taking the zero angular momentum limit $(a \rightarrow 0)$ of the Kerr results, the DeWitt-Schwinger approximation to the stress-energy in Schwarzschild may be found for an arbitrary collection of scalar, spinor, and vector fields. The resulting stress-energy tensor may again be decomposed into the contributions of the conformally coupled fields, $C_{\mu}^{\nu}$, and the contribution of a possibly nonconformal scalar field, $D_{\mu}^{\nu}$, according to Eq. (10). The components of the approximate stress-energy tensor for conformally coupled massive fields are

$$
\begin{aligned}
C_{T}^{T}=\frac{M^{2}}{1440 \pi^{2} T^{8}}\left\{\left[15-11\left(\frac{2 M}{T}\right)\right] \frac{1}{m_{0}^{2}}+\left[36-28\left(\frac{2 M}{T}\right)\right] \frac{1}{m_{1 / 2}^{2}}\right. \\
\left.+\left[-99+75\left(\frac{2 M}{T}\right)\right] \frac{1}{m_{1}^{2}}\right\} \\
C_{x}^{x}=\frac{M^{2}}{10080 \pi^{2} T^{8}}\left\{\left[-285+313\left(\frac{2 M}{T}\right)\right] \frac{1}{m_{0}^{2}}\right. \\
+\left[-540+596\left(\frac{2 M}{T}\right)\right] \frac{1}{m_{1 / 2}^{2}} \\
\left.+\left[1665-1833\left(\frac{2 M}{T}\right)\right] \frac{1}{m_{1}^{2}}\right\} \\
C_{\theta}^{\theta}=\frac{M^{2}}{10800 \pi^{2} T^{8}}\left\{\left[-315+367\left(\frac{2 M}{T}\right)\right] \frac{1}{m_{0}^{2}}\right. \\
+\left[-756+884\left(\frac{2 M}{T}\right)\right] \frac{1}{m_{1 / 2}^{2}} \\
\left.+\left[2079-2427\left(\frac{2 M}{T}\right)\right] \frac{1}{m_{1}^{2}}\right\}
\end{aligned}
$$

where $m_{0}, m_{1 / 2}$, and $m_{1}$ are the "effective masses" of the scalar, Dirac spinor, and vector fields present. If there is no field present for a particular spin, then its effective mass is set equal to infinity. If there are multiple fields with a given spin, possibly with differing masses (e.g., the massive spin$1 / 2$ fields in nature, representing the differing leptons and quarks), then the effective mass is calculated according to

$$
\frac{1}{m_{\mathrm{eff}}^{2}}=\sum_{i=1}^{n} \frac{1}{m_{i}^{2}}
$$

where the sum on the right-hand side is taken over the $n$ fields of given spin present.

The nonconformal scalar stress-energy contribution is given by

$$
\begin{aligned}
D_{T}^{T} & =\frac{M^{2}}{20 \pi^{2} m_{0}^{2} T^{8}}\left[-4+3\left(\frac{2 M}{T}\right)\right], \\
D_{x}^{x} & =\frac{M^{2}}{20 \pi^{2} m_{0}^{2} T^{8}}\left[10-11\left(\frac{2 M}{T}\right)\right], \\
D_{\theta}^{\theta} & =\frac{M^{2}}{10 \pi^{2} m_{0}^{2} T^{8}}\left[6-7\left(\frac{2 M}{T}\right)\right] .
\end{aligned}
$$

The DeWitt-Schwinger approximation for the stressenergy will be valid for sufficiently massive fields, when the Compton wavelength of the field, $\chi=\hbar / m$, is much smaller than the horizon radius of the black hole.

As was the case with the massless fields, these expressions show interesting behavior in the interior of the black hole. At the horizon, the energy density, $\rho=-\left\langle T_{T}^{T}\right\rangle$, is negative for all scalar fields with $\xi<2 / 9$, which includes the conformally and minimally coupled scalar fields. The spinor field has negative energy density at the horizon as well, whereas the vector field has positive energy density. As the singularity is approached the energy density diverges in a positive fashion for scalar fields with $\xi<47 / 216$, which again includes both the conformally and minimally coupled scalar fields. The energy density of the spinor field has a similar positive divergence, while the vector field energy density diverges negatively. Just as in the massless field case, the energy density of the scalar field is independent of the curvature coupling on the surface $T=3 M / 2$.

The spatial stress in the $x$-direction, $\left\langle T_{x}^{x}\right\rangle$, is positive on the horizon for all scalar fields with $\xi<2 / 9$, including the minimal and conformally coupled cases. As the singularity is approached, the stress shows a positive divergence for all scalar fields with $\xi<1237 / 5544$. For the spinor field, the spatial stress is also positive on the horizon and diverges in a positive direction as the singularity is approached. The vector field has negative stress in both limits.

The tangential stress, $\left\langle T_{\theta}^{\theta}\right\rangle$, is positive for all scalar fields with $\xi<55 / 252$, including the conformal scalar field. Again in this case, the stress for the spinor field is positive on the horizon and as the singularity is approached, and the vector field has negative stress in both cases.

\section{SEMICLASSICAL BLACK HOLE INTERIORS}

The linearized perturbations to the Schwarzschild metric resulting from the stress-energy of a quantized field (within the various analytic approximation schemes discussed in the previous section) have been described for the massless conformal scalar field by York [33], for the massless vector field by Hochberg and Kephart [34], and for the massless spinor field by Hochberg, Kephart, and York [35]. The perturbed geometry associated with a quantized massless scalar field with arbitrary curvature coupling has been analyzed by Anderson, Hiscock, Whitesell, and York [36]. In these previous calculations it was most convenient to describe the metric perturbations in ingoing Eddington-Finkelstein coordinates, $(v, r, \theta, \phi)$.

The study of the interior semiclassical effects proceeds most naturally however in terms of the original Schwarzschild coordinates (albeit with new names in the interior). In those coordinates, the perturbed metric may be written in the form

$$
\begin{aligned}
d s^{2}= & -\left(\frac{2 M}{T}-1\right)^{-1}[1+\epsilon \eta(T)] d T^{2}+\left(\frac{2 M}{T}-1\right) \\
& \times[1+\epsilon \sigma(T)] d x^{2}+T^{2} d \Omega^{2} .
\end{aligned}
$$

The Einstein equations, to first order in $\epsilon$, then have the form 


$$
\begin{aligned}
& \frac{d}{d T}[(2 M-T) \eta]=\frac{8 \pi T^{2}\left\langle T_{x}^{x}\right\rangle}{\epsilon}, \\
& \frac{d \sigma}{d T}=-\frac{8 \pi T^{2}\left\langle T_{T}^{T}\right\rangle}{\epsilon(2 M-T)}-\frac{\eta}{2 M-T} .
\end{aligned}
$$

\section{A. Massless fields}

Integrating Eqs. (37),(38) using the approximate stressenergy tensor for a collection of massless quantized fields given in Eqs. (11)-(28), one obtains

$$
\begin{aligned}
K \eta= & A\left[\left(\frac{T}{2 M}\right)^{2}+4\left(\frac{T}{2 M}\right)+12\left(1-\frac{T}{2 M}\right)^{-1} \ln \left(\frac{2 M}{T}\right)\right]+A_{0} \\
& +A_{1}\left(\frac{2 M}{T}\right)+A_{2}\left(\frac{2 M}{T}\right)^{2}+A_{3}\left(\frac{2 M}{T}\right)^{3} \\
K \sigma= & A\left[\left(\frac{T}{2 M}\right)^{2}+8\left(\frac{T}{2 M}\right)-24\left(\frac{3 M-T}{2 M-T}\right) \ln \left(\frac{2 M}{T}\right)\right]+B_{0} \\
& +B_{1}\left(\frac{2 M}{T}\right)+B_{2}\left(\frac{2 M}{T}\right)^{2}+B_{3}\left(\frac{2 M}{T}\right)^{3}
\end{aligned}
$$

where $K=3840 \pi$, and the coefficients $A_{i}, B_{i}$ are given by

$$
A=\frac{8 h(0)+7 h(1 / 2)+8 h(1)}{24},
$$

$$
\begin{gathered}
A_{0}=\frac{1}{24}[8(109-360 \xi) h(0)+43 h(1 / 2)+375 h(1)] \\
A_{1}=\frac{1}{24}[8(1-60 \xi) h(0)+67 h(1 / 2)-2872 h(1)], \\
A_{2}=\frac{1}{6}[8(-11+30 \xi) h(0)-17 h(1 / 2)-88 h(1)], \\
A_{3}=\frac{1}{24}[8(-83+300 \xi) h(0)-161 h(1 / 2)-664 h(1)]
\end{gathered}
$$

$$
B_{0}=\frac{1}{24}[8(155-720 \xi) h(0)+365 h(1 / 2)-565 h(1)]+k_{0},
$$

$$
B_{1}=\frac{1}{8}[8(-27+100 \xi) h(0)-89 h(1 / 2)+1064 h(1)],
$$

$$
B_{2}=\frac{1}{12}[8(-23+120 \xi) h(0)-41 h(1 / 2)+296 h(1)],
$$

and

$$
B_{3}=\frac{5}{24}[8(-5+36 \xi) h(0)+h(1 / 2)+152 h(1)]
$$

The form of Eq. (46) has been chosen so that the integration constant in $\sigma$ is expressed in terms of the integration constant, $k_{0}$, which has appeared in previous papers ${ }^{2}$ [3336]. The integration constant which is associated with $\eta$ has been absorbed via renormalization into $M$; the constant $M$ which appears in these equations is thus to be interpreted as the "dressed" mass of the black hole.

The semiclassical metric of Eq. (36) is valid only when the perturbations, $\epsilon \eta$ and $\epsilon \sigma$, are small compared to unity. The perturbations are small at the horizon, $T=2 M$, for black hole masses greater than or equal to the Planck mass (recall $\epsilon=\hbar / M^{2}=M_{P}^{2} / M^{2}$ ). Of course, the perturbations can always be made large by taking the large- $N$ limit, where $N$ is the number of quantized fields present. For reasonable numbers of fields, and black hole masses greater than the Planck mass, it is possible to approach the singularity at $T=0$ fairly closely. As an example, if we take $h(0)=0, h(1 / 2)=6$, $h(1)=2$, representing three massless neutrino fields and one massless vector field, and a black hole mass of $M=M_{P}$, then the perturbations reach a strength of $10^{-1}$ at about $T=M$; for a solar mass black hole, however, the perturbation does not reach this strength until $T \approx 3 \times 10^{-21}$ $\mathrm{cm}=2 \times 10^{-26} M$.

\section{B. Massive fields}

Integrating Eqs. (37),(38) using the approximate stressenergy tensor for a collection of massive quantized fields given in Eqs. (29)-(35), one obtains

$$
\begin{gathered}
K \eta=E\left[\left(\frac{2 M}{T}\right)+\left(\frac{2 M}{T}\right)^{2}+\left(\frac{2 M}{T}\right)^{3}+\left(\frac{2 M}{T}\right)^{4}+\left(\frac{2 M}{T}\right)^{5}\right] \\
+\widetilde{E}\left(\frac{2 M}{T}\right)^{6}, \\
K \sigma=k_{0}-E\left[-5+\left(\frac{2 M}{T}\right)+\left(\frac{2 M}{T}\right)^{2}+\left(\frac{2 M}{T}\right)^{3}\right. \\
\left.+\left(\frac{2 M}{T}\right)^{4}+\left(\frac{2 M}{T}\right)^{5}\right]+F\left[\left(\frac{2 M}{T}\right)^{6}-1\right]
\end{gathered}
$$

where $K$ is again equal to $3840 \pi$, and

$$
\begin{gathered}
E=\frac{1}{126 M^{2}}\left[(113-504 \xi) \frac{1}{m_{0}^{2}}+52 \frac{1}{m_{1 / 2}^{2}}-165 \frac{1}{m_{1}^{2}}\right], \\
\widetilde{E}=\frac{1}{126 M^{2}}\left[(-1237+5544 \xi) \frac{1}{m_{0}^{2}}-596 \frac{1}{m_{1 / 2}^{2}}+1833 \frac{1}{m_{1}^{2}}\right] \\
F=\frac{1}{18 M^{2}}\left[(-47+216 \xi) \frac{1}{m_{0}^{2}}-28 \frac{1}{m_{1 / 2}^{2}}+75 \frac{1}{m_{1}^{2}}\right]
\end{gathered}
$$

\footnotetext{
${ }^{2}$ In each of these papers the black hole was surrounded by a thin perfectly reflecting cavity. The specific value of the integration constant $k_{0}$ was obtained in those cases by requiring $g_{t t}$ to be continuous at the cavity wall. In the present work, none of our results will depend on the numerical value chosen for $k_{0}$.
} 
The integration constants in Eqs. (50),(51) are handled in the same manner as in the massless case; in particular, the black hole mass $M$ is the "dressed" or renormalized mass. The field masses $m_{0}, m_{1 / 2}, m_{1}$, are effective masses defined as described in Sec. III.

The perturbations of the Schwarzschild metric caused by the presence of massive fields are small, and the DeWittSchwinger approximation valid, so long as the Compton wavelength of the field is significantly less than the local radius of curvature of the spacetime. In the Schwarzschild interior, this will be true so long as $T \gg\left(M / m^{2}\right)^{1 / 3}$.

\section{ANISOTROPY OF THE SCHWARZSCHILD INTERIOR}

Since the Schwarzschild interior represents a highly anisotropic cosmology, it is natural to ask whether semiclassical effects dampen or strengthen the anisotropy. Many studies over the last quarter century have established that particle production can rapidly isotropize an anisotropic cosmology [3-11]. As mentioned in the introduction, the analytical approximations for massless fields are nonlocal and thus probably take particle production into account to some extent. However, it is completely unknown at this point how well they do this. The DeWitt-Schwinger approximation for the massive fields does not take particle production into account at all because it is a local approximation and particle production is an intrinsically nonlocal phenomenon. Thus whatever dissipation of anisotropy that is found due to all of these approximations is likely to be less that what would occur if full numerical solutions to the nonlinear backreaction equations were obtained.

One measure of the anisotropy of the interior is the ratio of the Hubble expansion rates in the differing spatial directions. In the present case, since the two spatial directions on the two-spheres of symmetry are equivalent, there is only one ratio to calculate, say

$$
\alpha=\frac{H_{x}}{H_{\theta}}=\frac{g_{\theta \theta} \frac{d g_{x x}}{d \tau}}{g_{x x} \frac{d g_{\theta \theta}}{d \tau}}=\frac{g_{\theta \theta} \frac{d g_{x x}}{d T}}{g_{x x} \frac{d g_{\theta \theta}}{d T}} .
$$

The sign of $\alpha$ is positive if the cosmology is expanding (or contracting) in all three spatial directions. If the cosmology is expanding or contracting isotropically, then $\alpha=1$.

Evaluating $\alpha$ for the metric of Eq. (36), we find, to first order in $\epsilon$

$$
\alpha=\alpha_{\mathrm{Sch}}+\epsilon \delta \alpha,
$$

where $\alpha_{\text {Sch }}$ is the ordinary Schwarzschild value,

$$
\alpha_{S c h}=\frac{-M}{2 M-T},
$$

and

$$
\delta \alpha=\frac{1}{2} T \frac{d \sigma}{d T}
$$

Taking Eq. (38) with Eq. (58), the perturbation to the anisotropy can be written explicitly in terms of components of the stress-energy as

$$
\delta \alpha=-\frac{4 \pi T}{\epsilon(2 M-T)}\left[T^{2}\left\langle T_{T}^{T}\right\rangle+\frac{1}{(2 M-T)} \int T^{2}\left\langle T_{x}^{x}\right\rangle d T\right] .
$$

If the overall sign of the perturbation to the anisotropy is positive, then the semiclassical effects tend to isotropize the interior. Negative values of $\delta \alpha$ push the spacetime towards greater anisotropy.

The interpretation of the semiclassical perturbations would be facilitated if expressions governing the physical effects such as that for $\delta \alpha$ in Eq. (59) could be understood solely in terms of the stress-energy properties such as positivity of the energy density. Unfortunately, inspection of Eq. (59) shows such a hope is in vain; not only is the perturbation in the anisotropy dependent on a mixture of stressenergy tensor and metric components, but since there is an integral in the expression for $\delta \alpha$, the anisotropy depends on the stress-energy in a nonlocal fashion.

Since the anisotropy is the ratio of the expansion rates along different spatial directions, careful consideration must be given to the method of spacetime slicing used to compare the perturbed and unperturbed spacetimes. One choice would be to consider slices which sit at equal proper times away from the horizon. Another choice, used in this paper, is to consider surfaces with equal values of the Schwarzschild area coordinate $T$.

Taking the stress-energy tensors described in the previous section for the quantized fields of interest, the contributions described in Eq. (59) can then be computed for various spin fields on the Schwarzschild background. It should be noted when considering these results that the perturbation expansions become less reliable as one proceeds away from the horizon and towards the singularity, but the exact point at which the perturbation should no longer be trusted is a matter of choice.

The perturbation to the anisotropy in the presence of a massless scalar field is

$$
\begin{aligned}
\delta \alpha= & \frac{1}{\pi(2 M-T)^{2}}\left\{M^{2}\left[\frac{\xi}{48}-\frac{17}{2880}\right]+\frac{M^{3}\left[\frac{\xi}{T}\left[\frac{7}{24}\right]\right.}{720}\right] \\
& +\frac{M^{4}}{T^{2}}\left[\frac{5 \xi}{12}-\frac{29}{720}\right]+\frac{M^{5}}{T^{3}}\left[\frac{5}{48}-\frac{3 \xi}{4}\right]+M T\left[\frac{29}{11520}-\frac{5 \xi}{192}\right. \\
& \left.\left.-\frac{\ln (2 M / T)}{960}\right]+\frac{T^{2}}{2304}+\frac{T^{3}}{11520 M}+\frac{T^{4}}{46080 M^{2}}\right\} .
\end{aligned}
$$

The sign of $\delta \alpha$ clearly depends on the value of the scalar curvature coupling, $\xi$. For values of $\xi<5 / 36$ the perturbation is positive, and the field tends to isotropize the spacetime. For values of $\xi>12 / 55$ the perturbation is negative and the spacetime tends to more anisotropy. Between these two values, $5 / 36<\xi<12 / 55$, the perturbation isotropizes in some regions of the interior and anisotropizes in other regions, as shown in Fig. 1. For values of $\xi$ and $T$ above the solid line, the spacetime is pushed towards anisotropy. Values of $\xi$ and $T$ below the solid line make $\delta \alpha>0$, and the spacetime is 


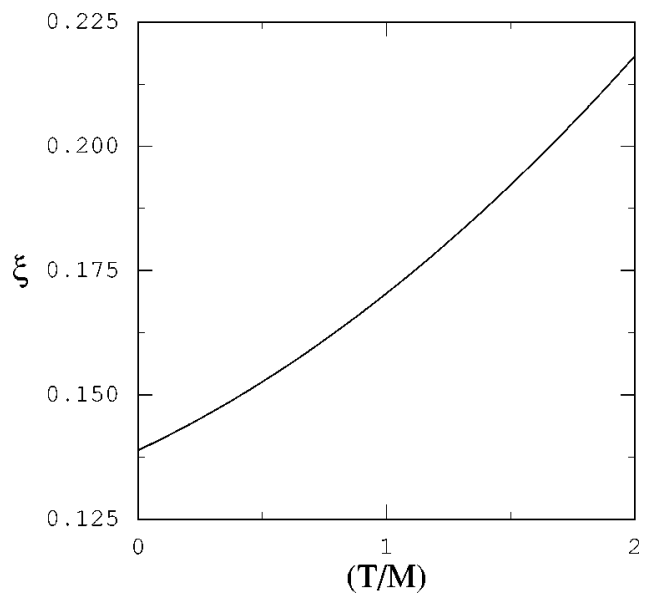

FIG. 1. The curve represents zero perturbation to the anisotropy of the interior for a massless scalar field as a function of the coordinate $T$ and the curvature coupling $\xi$. Above the curve, the perturbations due to the scalar field make the spacetime more anisotropic, and below the curve they make the spacetime more isotropic. As $T \rightarrow 0, \xi$ approaches $5 / 36$ on the curve. As $T \rightarrow 2 M, \xi$ approaches $12 / 55$.

isotropized in the presence of the scalar field. In this case, the minimally coupled scalar field $(\xi=0)$ always isotropizes the spacetime, whereas the conformally coupled field $(\xi=1 / 6)$ only isotropizes in the interior regions near the horizon.

The perturbation due to the massless spin-1/2 field is

$$
\begin{aligned}
\delta \alpha= & \frac{1}{\pi(2 M-T)^{2}}\left\{-\frac{M^{5}}{T^{3}} \frac{1}{192}+\frac{M^{4}}{T^{2}} \frac{97}{2880}-\frac{M^{3}}{T} \frac{19}{2880}\right. \\
& -M^{2} \frac{59}{11520}-M T\left[\frac{97}{46080}+\frac{7 \ln (2 M / T)}{3840}\right]+\frac{7 T^{2}}{9216} \\
& \left.+\frac{7 T^{3}}{46080 M}+\frac{7 T^{4}}{184320 M^{2}}\right\} .
\end{aligned}
$$

For $T>0.5$, which is the region where the perturbation expansion can be trusted, this always pushes the spacetime towards greater isotropy.

The massless vector field perturbation to the anisotropy is

$$
\begin{aligned}
\delta \alpha= & \frac{1}{\pi(2 M-T)^{2}}\left\{-\frac{19 M^{5}}{24 T^{3}}+\frac{211 M^{4}}{360 T^{2}}-\frac{97 M^{3}}{360 T}+\frac{343 M^{2}}{1440}\right. \\
& -M T\left[\frac{451}{5760}+\frac{\ln (2 M / T)}{480}\right]+\frac{T^{2}}{1152}+\frac{T^{3}}{5760 M} \\
& \left.+\frac{T^{4}}{23040 M^{2}}\right\}
\end{aligned}
$$

and pushes towards anisotropy for all values of $T$ in the interior.

The impact of massive fields of varying spin can be considered as well. For the massive scalar field

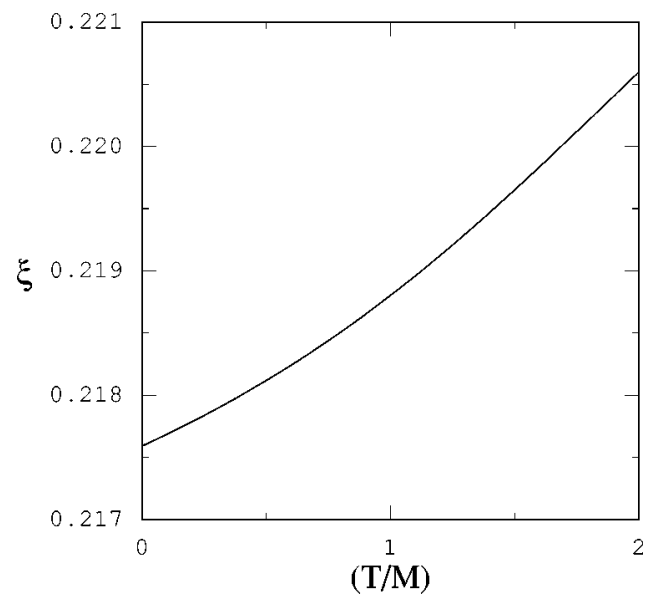

FIG. 2. The curve represents zero perturbation to the anisotropy of the interior for a massive scalar field as a function of the coordinate $T$ and the curvature coupling $\xi$. Above the curve, the perturbations make the spacetime more anisotropic, and below the curve they make the spacetime more isotropic. As $T \rightarrow 0, \xi$ approaches $47 / 216$ on the curve. As $T \rightarrow 2 M, \xi$ approaches $1223 / 5544$.

$$
\begin{aligned}
\delta \alpha= & \frac{1}{\pi m^{2}}\left(\frac{M^{4}}{T^{6}}\left[\frac{47}{360}-\frac{3 \xi}{5}\right]+\frac{M^{3}}{T^{5}}\left[\frac{113}{6048}-\frac{\xi}{12}\right]\right. \\
& +\frac{M^{2}}{T^{4}}\left[\frac{113}{15120}-\frac{\xi}{240}\right]+\frac{M}{T^{3}}\left[\frac{113}{40320}-\frac{\xi}{80}\right] \\
& \left.+\frac{1}{T^{2}}\left[\frac{113}{120960}-\frac{\xi}{240}\right]+\frac{1}{M T}\left[\frac{113}{483840}-\frac{\xi}{960}\right]\right\},
\end{aligned}
$$

where $m$ is the effective field mass defined in Eq. (32). Similar to the case of the massless scalar field, the exact sign of the perturbation depends on the value of the scalar curvature coupling. When $\xi>1223 / 5544$, the presence of the field makes the spacetime more anisotropic, and when $\xi<47 / 216$ the push is always towards isotropy. As shown in Fig. 2, there exists a range of values $47 / 216<\xi<1223 / 5544$ over which some interior regions are isotropized and others are not. As before, values of $\xi$ and $T$ above the solid line have $\delta \alpha<0$ and the spacetime tends towards anisotropy. For values below the solid line, $\delta \alpha>0$, and the tendency is towards isotropy. Both minimal and conformal coupling fall within this regime.

The perturbation due to a massive spinor field is

$$
\begin{aligned}
\delta \alpha= & \frac{1}{\pi m^{2}}\left\{\frac{M^{4}}{T^{6}} \frac{7}{90}+\frac{M^{3}}{T^{5}} \frac{113}{1512}+\frac{M^{2}}{T^{4}} \frac{13}{3780}+\frac{M}{T^{3}} \frac{13}{10080}\right. \\
& \left.+\frac{1}{T^{2}} \frac{13}{30240}+\frac{1}{M T} \frac{13}{120960}\right\},
\end{aligned}
$$

which is manifestly positive for all values of $T$, and hence decreases the anisotropy.

Similarly, the massive vector field perturbation to the anisotropy is 


$$
\begin{aligned}
\delta \alpha= & -\frac{1}{\pi m^{2}}\left\{\frac{M^{4}}{T^{6}} \frac{5}{24}+\frac{M^{3}}{T^{5}} \frac{55}{2016}+\frac{M^{2}}{T^{4}} \frac{11}{1008}+\frac{M}{T^{3}} \frac{11}{2688}\right. \\
& \left.+\frac{1}{T^{2}} \frac{11}{8064}+\frac{1}{M T} \frac{11}{32256}\right\},
\end{aligned}
$$

which is manifestly negative for all $T$, and so always tends to increase the anisotropy.

\section{APPROACHING THE FINAL SINGULARITY}

Ever since it was realized that singularities could not be avoided in physically plausible spacetimes, quantum effects have been invoked as the physical instrument which might restore regularity to spacetime, by banishing singular behavior. While it is impossible for a perturbative analysis to determine whether quantum effects will eradicate (or strengthen) the singularity, it is possible to determine how the growth of curvature as one approaches the singularity is initially affected by the semiclassical perturbation, before the semiclassical correction grows so large that the perturbative approach becomes invalid.

The simplest way to see the effect of quantized fields on the growth of curvature as one approaches the singularity is to examine the perturbations of curvature scalars. One such scalar is the Kretschmann scalar, which for unperturbed Schwarzschild is

$$
K_{\mathrm{Sch}}=R^{\alpha \beta \mu \nu} R_{\alpha \beta \mu \nu}=\frac{48 M^{2}}{T^{6}} .
$$

The Kretschmann scalar is perfectly well behaved near the horizon $T=2 M$, but diverges strongly, as $T^{-6}$, as $T \rightarrow 0$.

Evaluating $K$ to first order in $\epsilon$ for the metric of Eq. (36) will yield

$$
K=K_{\mathrm{Sch}}+\epsilon \delta K
$$

The first order correction to the Kretschmann scalar can be written in terms of the perturbation functions $\eta$ and $\sigma$ as

$$
\begin{aligned}
\delta K= & \frac{8}{T^{3}}\left[-12 \eta \frac{M^{2}}{T^{3}}+2 \eta \frac{M}{T^{2}}+3 \eta^{\prime} \frac{M^{2}}{T^{2}}-\eta^{\prime} \frac{M}{T}-5 \sigma^{\prime} \frac{M^{2}}{T^{2}}\right. \\
& \left.+\sigma^{\prime} \frac{M}{T}+2 \sigma^{\prime \prime} \frac{M^{2}}{T}-\sigma^{\prime \prime} M\right]
\end{aligned}
$$

where primes denote differentiation with respect to $T$.

In general the perturbation to the Kretschmann scalar diverges more strongly that the classical scalar, namely as $T^{-9}$ for massless field perturbations and as $T^{-12}$ for massive field perturbations. On this basis, one might expect that quantum effects always increase the rate of curvature growth, strengthening the singularity. However, it must be kept in mind that the perturbed metric and curvature are only valid in the region where the deviation from the classical Schwarzschild metric is small. Precisely where (for what value of $T$ ) the perturbed metric becomes a poor approximation to a full self-consistent solution depends on a number of factors, including the mass of the black hole. It is clear, however, that the perturbed metric can never be valid all the way down to the singularity at $T=0$. Thus, questions about

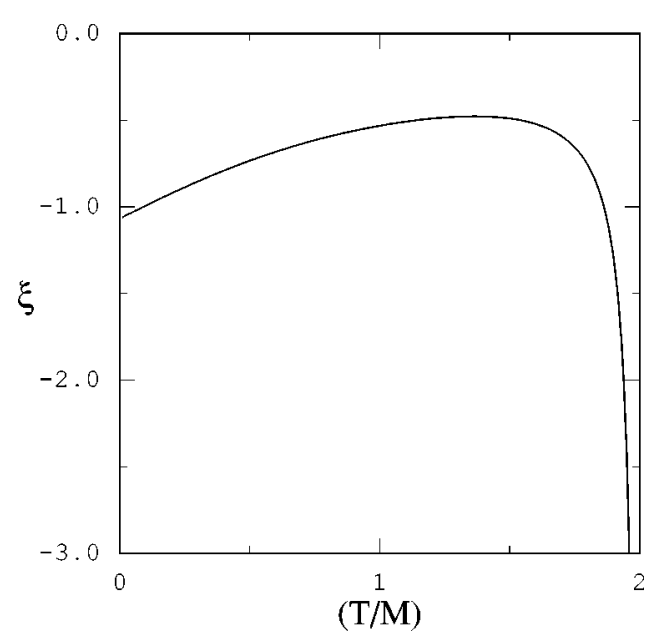

FIG. 3. The curve represents zero perturbation to the Kretschmann scalar for a massless scalar field as a function of the coordinate $T$ and the curvature coupling $\xi$. Above the curve, the perturbations are positive, increasing the rate of curvature growth, and below the curve the perturbations are negative, decreasing the growth of curvature relative to the classical solution.

whether the singularity is ultimately strengthened or weakened, or even removed, by quantum effects cannot be answered definitively within the perturbative approach employed here.

In order to gain insight into whether the quantum effects associated with the different fields would tend to strengthen or weaken the singularity, we examine the direction in which the perturbed Kretschmann scalar initially deviates from the classical value, at large $T$, where the perturbed metric is most valid. If the sign of $\delta K$ is positive, the initial rate of growth of curvature as one approaches the singularity will be strengthened. If $\delta K$ is negative, then the initial rate of growth of curvature will be weakened when compared to the classical metric. We consider the sign of the perturbation for all possible values of $T$, since one cannot say that the perturbation becomes invalid at a particular value of $T$ without additional information about a particular black hole. However, our results are certainly only valid in the domain where $|\delta K| \ll|K|$.

The perturbation to $K_{\text {Sch }}$ in the presence of a massless scalar field is

$$
\begin{aligned}
\delta K= & \frac{768 \pi}{\lambda T^{4}}\left\{\frac{M^{5}}{T^{5}}[2048+1920 \xi]-\frac{M^{4}}{T^{4}}[400-960 \xi]\right. \\
& -\frac{M^{3}}{T^{3}}[240-480 \xi]+\frac{M^{2}}{T^{2}}[16-720 \xi-48 \ln (2 M / T)] \\
& \left.+12 \frac{M}{T}+1\right\} .
\end{aligned}
$$

The sign of $\delta K$ depends on the value of the scalar curvature coupling, $\xi$. Figure 3 shows a plot of the curvature coupling $\xi$ vs $T$ over the interior. The solid line represents values of $\xi$ and $T$ for which $\delta K=0$. For points below the solid line, the perturbation to the Kretschmann scalar is negative, and for values above the line the perturbation is positive. For all 
non-negative values of the curvature coupling, the contribution is positive, and hence curvature grows faster than in the unperturbed metric.

The massless spinor field perturbs $K_{\text {Sch }}$ by

$$
\begin{aligned}
\delta K= & \frac{1344 \pi}{\lambda T^{4}}\left\{\frac{9536}{7} \frac{M^{5}}{T^{5}}-\frac{3120}{7} \frac{M^{4}}{T^{4}}-\frac{1760}{7} \frac{M^{3}}{T^{3}}\right. \\
& \left.-\frac{M^{2}}{T^{2}}\left[\frac{368}{7}+\frac{336}{7} \ln (2 M / T)\right]+\frac{84}{7} \frac{M}{T}+1\right\} .
\end{aligned}
$$

The perturbation of Eq. (70) changes sign in the interior, yielding a negative contribution to the Kretschmann scalar for $T>1.479 M$, and a positive contribution to the Kretschmann scalar for $T<1.479 M$. Whether the perturbation strengthens or weakens the growth of curvature thus depends on the value of $T$ at which the perturbed metric becomes unreliable.

The massless vector field perturbation to the Kretschmann scalar is

$$
\begin{aligned}
\delta K= & \frac{1536 \pi}{\lambda T^{4}}\left\{14528 \frac{M^{5}}{T^{5}}-1680 \frac{M^{4}}{T^{4}}+4960 \frac{M^{3}}{T^{3}}\right. \\
& \left.-\frac{M^{2}}{T^{2}}[1664+48 \ln (2 M / T)]+12 \frac{M}{T}+1\right\},
\end{aligned}
$$

which is positive for all values of $T$ in the interior; the massless vector field thus seems to strengthen the growth of curvature as the singularity is approached.

Similar considerations can be given to massive fields. In the case of the massive scalar field

$$
\begin{aligned}
\delta K= & \frac{1}{5040 \pi m^{2} T^{6}}\left\{-\frac{M^{6}}{T^{6}}[2112-32256 \xi]\right. \\
& \left.-2304 \frac{M^{5}}{T^{5}}+113-504 \xi\right\} .
\end{aligned}
$$

As in the massless scalar field case, the exact sign of the perturbation depends on the scalar curvature coupling. Figure 4 shows a plot of the curvature coupling $\xi$ vs $T$ over the interior. Values of $\xi$ and $T$ below the solid line yield $\delta K<0$. For points above the solid line, $\delta K>0$. The minimally coupled massive scalar field weakens the growth of curvature over most of the interior, but near the horizon, for $T>1.973 M$, the curvature is strengthened. For the conformally coupled scalar field, the rate of curvature growth is always greater than in the unperturbed Schwarzschild metric.

For the massive spinor field

$$
\delta K=\frac{1}{1260 \pi m^{2} T^{6}}\left\{-5952 \frac{M^{6}}{T^{6}}+2304 \frac{M^{5}}{T^{5}}+13\right\},
$$

which is negative for all interior values of the coordinate $T$; hence the massive spinor field softens the approach to the singularity, decreasing the rate of increase of the curvature.

In contrast, the massive vector field has

$$
\delta K=\frac{1}{1680 \pi m^{2} T^{6}}\left\{8640 \frac{M^{6}}{T^{6}}-2304 \frac{M^{5}}{T^{5}}-55\right\},
$$

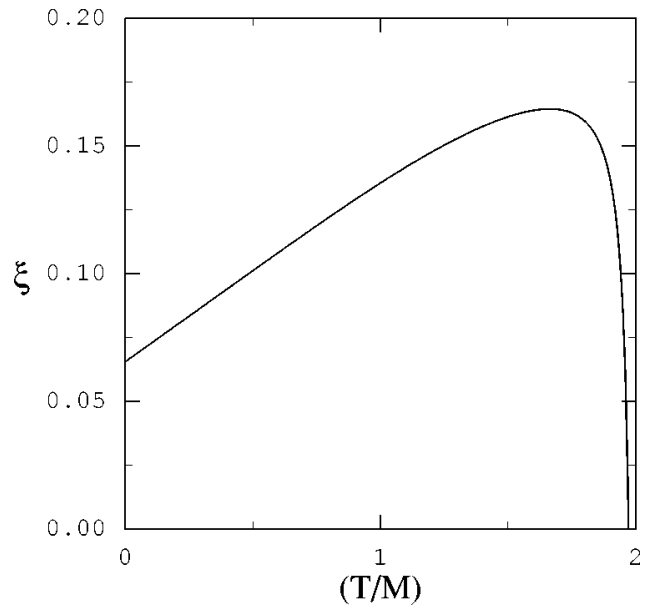

FIG. 4. The curve represents zero perturbation to the Kretschmann scalar for a massive scalar field as a function of the coordinate $T$ and the curvature coupling $\xi$. Above the curve, the perturbations to Kretschmann are positive, and below the curve the perturbations are negative.

which is positive for all values of $T$ in the interior; hence the massive vector field strengthens the growth of curvature as the singularity is approached.

\section{DISCUSSION AND SUMMARY}

In this paper we have calculated the linearized perturbations of the Schwarzschild black hole interior due to a collection of quantized matter fields. The stress-energy tensor of the matter fields has been described using analytic approximations. For massless fields, we have used the approximations of Page, Brown, and Ottewill [20] for the spinor field, the approximation of Jensen and Ottewill [28] for the vector field, and that of Anderson, Hiscock, and Samuel [22] for the scalar field. Massive fields have been treated using the DeWitt-Schwinger approximation, as developed by Frolov and Zel'nikov [24] and Anderson, Hiscock, and Samuel [22].

These calculations provide virtually all of the useful information about semiclassical effects in the interior of a black hole that can be obtained using the various analytical approximations. One could attempt to construct fully selfconsistent solutions to the semiclassical equations using the DeWitt-Schwinger approximation for massive fields or the approximations of Frolov and Zel'nikov [21] or Anderson, Hiscock, and Samuel [22] for massless fields. However, serious problems arise in such calculations. For massless fields the analytical approximations diverge logarithmically on the event horizon in any static non-Ricci-flat spacetime. Numerical computations of the stress-energy tensor in ReissnerNordström spacetimes [22,29] indicate that these divergences are not real. They are simply an indication that it is only for Schwarzschild spacetime that the analytical approximations are valid near the event horizon. For massive fields the DeWitt-Schwinger approximation gives no divergent behavior on the event horizon of any black hole. However, this approximation is valid only in the limit that the Compton wavelength of the field is much smaller than the radius of curvature of the spacetime. Thus the best that can be done when using the DeWitt-Schwinger approximation is to solve the semiclassical equations perturbatively, in which case the 
first order term is definitely the most important. Therefore, it will be necessary to numerically compute the stress-energy tensor to study semiclassical interior effects beyond the level of linear perturbation theory.

We have addressed the question of whether anisotropy is dissipated in the interior by treating the black hole interior as an anisotropic, homogeneous cosmology and examining whether the perturbed metric has greater or lesser anisotropy than the background Schwarzschild metric. We find that minimally and conformally coupled scalar fields, and the spinor field, decrease the anisotropy as one approaches the singularity, while vector fields increase the anisotropy. These results are described from the point of view of the black hole interior, which as a cosmology is a universe approaching a final singularity. If one instead interpreted our results in terms of the white hole portion of the Schwarzschild Penrose diagram, then scalar and spinor fields would enhance anisotropy as one moves away from the singularity, while vector fields would reduce it. However as previously mentioned, in this case the boundary conditions for the fields are "final" rather than "initial" conditions.

We have also examined whether there is any evidence for the semiclassical perturbation modifying the approach to the singularity. While in all cases the perturbed curvature ultimately diverges more strongly than in the classical Schwarzschild metric, the perturbed metric becomes an invalid approximation to the true semiclassical metric long before that occurs. Accordingly, within the context of perturbation theory, it is impossible to determine whether quantum effects might substantially change the character of the singularity (perhaps even eliminating it). One can, however, ask whether an observer approaching the final black hole singularity will measure larger or smaller curvature in the perturbed metric than in the classical Schwarzschild case. We find that massless fields of all spin, the conformally coupled massive scalar and massive vector fields, generally strengthen the singularity (curvature grows faster than in Schwarzschild) while the massive minimally coupled scalar and spinor fields weaken the growth of curvature in the domain where the perturbed metric is valid.

\section{ACKNOWLEDGMENTS}

This work was supported in part by NSF Grants Nos. PHY92-07903 and PHY95-11794 (W.A.H.), and PHY9512686 (P.R.A.).
[1] S. W. Hawking, Commun. Math. Phys. 43, 199 (1975).

[2] See, for example, G. W. Gibbons, in General Relativity: An Einstein Centenary Survey, edited by S. W. Hawking and W. Israel (Cambridge University Press, Cambridge, England, 1979), and references contained therein.

[3] Y. B. Zel'dovich, Pis'ma Zh. Éksp. Teor. Fiz. 12, 307 (1970) [JETP Lett. 12, 307 (1970)].

[4] Y. B. Zel'dovich and A. A. Starobinsky, Zh. Éksp. Teor. Fiz. 61, 2161 (1971) [Sov. Phys. JETP 34, 1159 (1971)].

[5] B. L. Hu, Phys. Rev. D 8, 1048 (1973); 9, 3263 (1974).

[6] B. L. Hu, S. A. Fulling, and L. Parker, Phys. Rev. D 8, 2377 (1973).

[7] S. A. Fulling, L. Parker, and B. L. Hu, Phys. Rev. D 10, 3905 (1974); 11, 1714(E) (1975).

[8] B. Berger, Ann. Phys. (N.Y.) 83, 458 (1974); Phys. Rev. D 11, 2770 (1975); 12, 368 (1975).

[9] V. N. Lukash and A. A. Starobinsky, Zh. Éksp. Teor. Fiz. 66, 1515 (1974) [Sov. Phys. JETP 32, 742 (1974)].

[10] B. L. Hu and L. Parker, Phys. Rev. D 17, 933 (1978).

[11] J. B. Hartle and B. L. Hu, Phys. Rev. D 20, 1772 (1979).

[12] L. Parker and S. Fulling, Phys. Rev. D 7, 2357 (1973).

[13] A. A. Starobinsky, Phys. Lett. 91B, 99 (1980).

[14] M. V. Fischetti, J. B. Hartle, and B. L. Hu, Phys. Rev. D 20, 1757 (1979).

[15] P. Anderson, Phys. Rev. D 28, 271 (1983); 29, 615 (1984).

[16] T. Azuma and S. Wada, Prog. Theor. Phys. 75, 845 (1986).

[17] R. Kantowski and R. K. Sachs, J. Math. Phys. (N.Y.) 7, 443 (1966).

[18] D. N. Page, Phys. Rev. D 25, 1499 (1982).
[19] M. R. Brown and A. C. Ottewill, Phys. Rev. D 31, 2514 (1985).

[20] M. R. Brown, A. C. Ottewill, and D. N. Page, Phys. Rev. D 33, 2840 (1986).

[21] V. P. Frolov and A. I. Zel'nikov, Phys. Rev. D 35, 3031 (1987).

[22] P. R. Anderson, W. A. Hiscock, and D. A. Samuel, Phys. Rev. Lett. 70, 1739 (1993); Phys. Rev. D 51, 4337 (1995).

[23] V. P. Frolov and A. I. Zel'nikov, Phys. Lett. 115B, 372 (1982).

[24] V. P. Frolov and A. I. Zel'nikov, Phys. Rev. D 29, 1057 (1984).

[25] R. Herman and W. A. Hiscock, report 1997 (unpublished).

[26] K. W. Howard and P. Candelas, Phys. Rev. Lett. 53, 403 (1984).

[27] K. W. Howard, Phys. Rev. D 30, 2532 (1984).

[28] B. P. Jensen and A. Ottewill, Phys. Rev. D 39, 1130 (1989).

[29] P. R. Anderson, W. A. Hiscock, and D. J. Loranz, Phys. Rev. Lett. 74, 4365 (1995).

[30] S. Christensen, Phys. Rev. D 14, 2490 (1976).

[31] P. Candelas and B. P. Jensen, Phys. Rev. D 33, 1596 (1986).

[32] B. S. DeWitt, Dynamical Theory of Groups and Fields (Gordon and Breach, New York, 1965).

[33] J. W. York, Jr., Phys. Rev. D 31, 775 (1985).

[34] D. Hochberg and T. W. Kephart, Phys. Rev. D 47, 1465 (1993).

[35] D. Hochberg, T. W. Kephart, and J. W. York, Jr., Phys. Rev. D 48, 479 (1993).

[36] P. R. Anderson, W. A. Hiscock, J. Whitesell, and J. W. York, Jr., Phys. Rev. D 50, 6427 (1994). 